\section{Vietnam Journal of Agricultural Sciences}

\title{
Prophylactic and Therapeutic Methods Against Histomoniasis in Poultry
}

\author{
Nguyen Van Phuong \& Bui Khanh Linh
}

Faculty of Veterinary Medicine, Vietnam National University of Agriculture, Hanoi 131000, Vietnam

\begin{abstract}
Histomoniasis, commonly known as blackhead disease, is a parasitic disease in poultry caused by the protozoan Histomonas meleagridis. The availability of various compounds for chemotherapy in the 1970s resulted in the successful control of blackhead disease. Since the ban of antihistomonal drugs in the European Union, the disease has reemerged, resulting in up to $100 \%$ mortality in turkey flocks. This has renewed the interest of scientists with numerous publications focusing on prophylactic strategies. This review summarizes the literature on the preventive and curative options for the control and treatment of histomoniasis. Two main approaches to the prophylaxis of the disease were found, which included chemotherapies and plant substrate products. Histostat-50 and paromomycin were the only available drugs that showed antihistomonal activity despite some concern about their threat to human health and antibiotic resistance. None of the plant substrate products provided potential protection to birds against blackhead disease. The use of attenuated histomonads could be an alternative for the prevention of the disease, but the production of this vaccine prototype is still challenging due to advanced technique requirements.
\end{abstract}

\section{Keywords}

Histomoniasis, Histomonas meleagridis, parasitic in poultry, prophylactic, therapeutic

\section{Historical treatment options}

Histomoniasis, also known as blackhead disease, histomonosis, or infectious enterohepatitis, is a parasitic disease caused by the protozoan Histomonas meleagridis, and was first described by

Received: May 15, 2020 Accepted: July 20, 2020

\section{Correspondence to} phuongnv@vnua.edu.vn

\section{ORCID}

Nguyen Van Phuong https://orcid.org/0000-0003-25296891 Cushman (1893). The disease can cause inflammatory lesions in the caeca and liver of gallinaceous birds, and may lead to flock mortality of $80-100 \%$ in turkey flocks (Swayne et al., 2013). Although apparent clinical signs are not observed in infected chickens, the disease can also cause significant mortality in this host (Reece et al., 1986).

Since the 1970s, histomoniasis has been successfully controlled 
by using antiflagellate feed additives such as dimetridazole and nifursol (Hafez et al., 2005). In subsequent years, very little was published on the prevention and treatment of the disease (Figure 1). Between the 1990s and 2003, the European Union successively banned all preventive and curative drugs against $H$. meleagridis for use in food-producing animals because of public concerns about toxicity and carcinogenicity of the antihistomonal drugs (Byrne, 2001; Hafez et al., 2005). As a result of the removal of these treatments, there have been numerous outbreaks of histomoniasis in the layer, free-range chicken and turkey flocks, which have caused considerable economic losses. A case that demonstrated such losses was reported in Germany where an outbreak occurred in a free-range layer flock of hens resulting in $6 \%$ increased mortality and $11 \%$ decreased egg production (Esquenet et al., 2003). Between 2004 and 2008, about 65 flocks in Germany were infected with $H$. meleagridis. In 10 farms of commercial turkeys, a total of 144,000 birds were detected as infected with the mortality rate rising by $40 \%$ within one week (Hafez et al., 2005; Hauck et al., 2010).

Outbreaks occur not only in such developed countries but have also been noticed in some developing countries including Thailand, Vietnam, and Brazil (Somkid \& Niwat, 2006; Nguyen et al., 2015; Araújo et al., 2015). A mortality of $70-80 \%$ was recorded in an outbreak in Thailand in 2006. In 2015, an outbreak of histomoniasis in free-range chickens with a mortality of $43.7 \%$ was reported in Brazil (Araújo et al., 2015). The standards for drug usage differ between regions, and in many cases, drugs are used without sufficient scientific evidence of their efficacy.

Another factor complicating the treatment of histomoniasis was the finding that a strain of $H$. meleagridis was partially resistant to nitarsone, the only approved prophylactic antihistomonal in the U.S. (Abraham et al., 2014). Additionally, arsenical residue was recently found in the livers of roxarsone-treated chickens resulting in the withdrawal of this substance from the U.S. market (Kawalek et al., 2011).

This situation has led $H$. meleagridis to become of renewed scientific interest, with a number of recent studies (Figure 1) focusing on different prophylactic strategies including the use of chemotherapy drugs such as arsenical, synthesized nitroheterocylic compounds, and anthelmintics (van der Heijden, 2009). Plant substances have also become an alternative treatment of this parasitic disease. Among all essential oils and extracts, Natustat and Protophyt are two commercially available plant products that have been tested as possible comments. We acknowledge the insightful

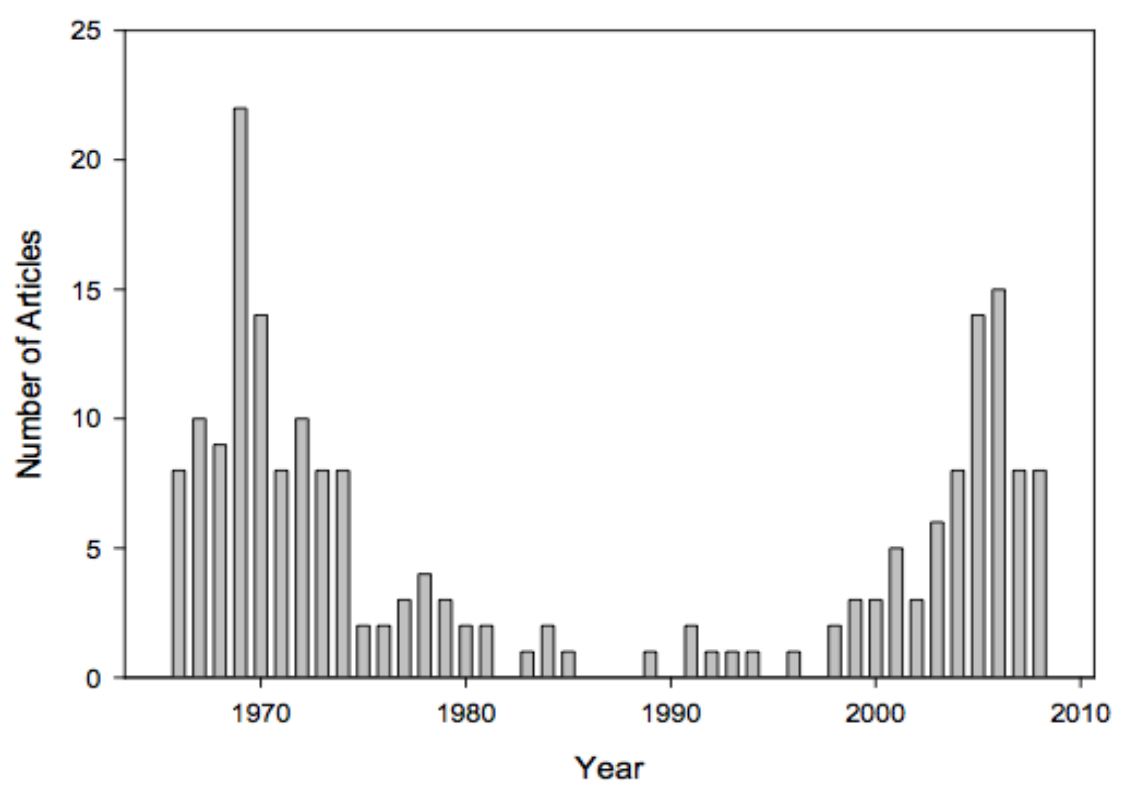

Figure 1. Number of scientific articles on H. meleagridis per year during the last four decades (van der Heijden, 2009) 
treatments for histomoniasis (Duffy et al., 2004). Focusing on immunizations against the disease, researchers have attempted vaccines with attenuated cultures of $H$. meleagridis in vitro and in vivo. The focus of this review is on prophylactic and therapeutic options for the control and treatment of histomoniasis in birds including chemotherapeutic drugs, plant substrate products, and vaccinations in the U.S. and the E.U.

\section{Current treatment options}

\section{Chemotherapies}

Arsenical compounds have been known to be very effective against histomonad infection by reducing mortality. However, the concern over arsenic toxicity resulted in the banning of these chemotherapies in the European Union in the period from the 1990s to 2003 (Byrne, 2001). Following the withdrawal of these compounds, scientists sought to find new drugs with antihistomonal properties and began the testing of various recognized antiprotozoal drugs.

In an in vitro experiment realized by $\mathrm{Hu}$ and McDougald (2004), nine antiprotozoal drugs were tested for activity against $H$. meleagridis, namely tinidazole, metronidazole, ornidazole, and diloxanide furoate, which are known as compounds active against amoebas and flagellates; albendazole and mebendazole, which are anthelmintics with antiprotozoal properties; and also some antibiotics including paromomycin sulfate, quinoline, and carbadox. Dimetridazole, an effective antihistomonal drug, which was previously banned in 2003 (Byrne, 2001) was used as a control in the study. In the in vitro experiment, a dose of 500,000 histomonads $\mathrm{mL}^{-1}$ was cultured in a modified Dwyer medium with different concentrations of the tested drugs. The results showed that growth of $H$. meleagridis was completely inhibited at $10 \mu \mathrm{g} \mathrm{mL}^{-1}$ or higher of dimetridazole, ornidazole, tinidazole, and metronidazole. Paromomycin sulfate only suppressed the growth of the parasite at $100 \mu \mathrm{g}$ $\mathrm{mL}^{-1}$ or higher, while other drugs did not show any antihistomonal effects. These results led to an in vivo test where chickens, intracloacally inoculated with 200,000 H. meleagridis, were subjected to the successfully tested drugs in the in vitro test. Dimetridazole, metronidazole, ornidazole, and tinidazole showed their high antihistomonal activity at $200-400 \mathrm{ppm}$, while paromomycin sulfate and carbadox neither improved weight gain nor reduced liver and caecal lesion scores (Hu \& McDougald, 2004) These findings were quite persuasive since the authors used a strong study design where an in vitro experiment was followed by an in vivo experiment for confirmation. The sample size used in this study was sufficiently reliable $(\mathrm{n}=$ 360 birds). Moreover, the dose of $H$. meleagridis and the intracloacal inoculation has successfully been applied by previous researchers on various occasions (McDougald \& $\mathrm{Hu}, 2001$; Grabensteiner et al., 2006; Zahoor et al., 2011) which made the findings of this study more reliable.

In subsequent years, the prophylactic effect of paromomycin against histomoniasis was tested in turkeys (Hafez et al., 2010). Similar to the methods of $\mathrm{Hu}$ and McDougald (2004), turkeys were challenged with $H$. meleagridis by intracloacal infection and then treated with different concentrations of paromomycin via a feed additive. These researchers found that the birds treated with paromomycin had significantly reduced mortality, reduced liver and caecal lesion scores, and an increased feed conversion rate compared to the control birds, indicating that paromomycin had exhibited its antihistomonal efficacy (Hafez et al., 2010). This is in line with the results of a study conducted by Bleyen et al. (2009) where paromomycin, both in the commercial form of histoBloc and in drinking water, showed its preventative effect in $10,000 \mathrm{H}$. meleagridis intracloacally infected turkeys. However, this drug did not demonstrate a curative effect due to the aminoglycoside nature of paromomycin, which did not allow the drug to reach the liver stage (Bleyen et al., 2009). Recently, van de Heijden et al. (2011) confirmed the effect of this antibiotic in the prevention of blackhead disease. Both in vitro and in vivo experiments were carried out with 3 different concentrations of paromomycin ranging from 100 to $400 \mathrm{ppm}$. Although this antibiotic suppressed the growth of histomonads at all 
concentrations under in vitro conditions, only the highest concentration provided full protection to challenged poults (Van de Heijden et al., 2011). The high dose recommended in this study was not supported by the European Food Safety Authority due to the concern of bacterial resistance (EFSA, 2009). Kempf et al. (2013) proved the antimicrobial resistance of intestinal bacteria to paromomycin supplementation in feed additives in turkeys. Escherichia coli, Enterococcus faecium, and Staphylococcus aureus became resistant to paromomycin and other aminoglycosides in turkeys fed with paromomycin in feed additives. In addition, $E$. coli and E. faecium showed a co-selection for resistance to other antibiotics (Kempf et al., 2013).

In essence, paromomycin has been identified as the most effective antihistomonal drug among various tested antibiotics despite the concern that a high dose of this drug could cause intestinal bacterial resistance in supplemented birds.

Tiamulin was another antibiotic of interest for scientists during the search for new drugs effective against blackhead disease. The curative effect of this drug was reported for the first time by Burch, who treated infected turkeys with tiamulin via drinking water at $25 \mathrm{mg} \mathrm{kg}^{-1}$ body weight for five days (van der Heijden et al., 2011). In this case, mortality was reduced significantly, and subsequently, the histomoniasis outbreak was controlled. According to this report, tiamulin had a therapeutic effect on blackhead disease, which shows promise that it could potentially be a new approach to the treatment of the disease. These findings, however, would be more convincing if the authors carried out a thorough microscopical diagnosis of histomoniasis or prepared a parasite culture to confirm the presence of $H$. meleagridis since caecal necrosis could be a result of coccidiosis (EFSA, 2009). In addition, it was likely that the authors ignored the co-effect of amoxicillin that birds had been previously supplied. Therefore, it would be beneficial to perform an in vivo experiment to confirm the antihistomonal effect of this antibiotic.

Hauck et al. (2010) carried out in vitro experiments in which the antihistomonal activity of tiamulin was assessed. Tiamulin was tested at different concentrations ranging from 20 to 320 $\mathrm{mg} \mathrm{L}^{-1}$. In the experiments, different strains of $H$. meleagridis including strains 542/04, 551/04, and 1169/09 were used in different inoculation doses from 10,000 to 40,000 parasites $\mathrm{mL}^{-1}$. In this study, tiamulin showed its inhibitory activity to histomonal growth at all tested concentrations. Furthermore, this antibiotic, at all concentrations, induced lethality to exposed histomonads of the low dose of inoculation while a high concentration $\left(320 \mathrm{mg} \mathrm{L}^{-1}\right)$ of this antibiotic could completely destroy parasites at even a high dose $\left(40,000\right.$ parasites $\left.\mathrm{mL}^{-1}\right)$. This study demonstrated that tiamulin was effective against $H$. meleagridis in vitro. This finding, however, is not convincing since the doses of inoculation used in this study were relatively lower than those used in other studies. The highest dose of 40,000 histomonads $\mathrm{mL}^{-1}$ was lower than 60,000 used in the study of van der Heijden (2009), and much lower than 500,000 in the experiment of $\mathrm{Hu}$ \& McDougald (2004). Moreover, in this study, the authors pointed out that the effectiveness of the tested drug decreased when the dose of inoculation increased. Therefore, the effect of tiamulin would be questionable with a dose of inoculation as high as used in other studies.

A year later, van der Heijden et al. (2011) performed another in vitro experiment to evaluate the antihistomonal activity of tiamulin. Two-fold serial dilutions ranging from 2.5 to 400 $\mu \mathrm{g} \mathrm{mL} \mathrm{mL}^{-1}$ of the antibiotic in PBS were added to cultures of the parasite. The starting concentration of histomonads used in the cultures was 550,000 parasites $\mathrm{mL}^{-1}$. The results obtained from this experiment showed that the highest concentration of tiamulin $\left(400 \mu \mathrm{g} \mathrm{mL}^{-1}\right)$ induced a slightly lower number of histomonads in the culture compared to the negative control cultures. This indicated that the antibiotic was unable to inhibit the growth of histomonads in the culture environment. The difference between the results of this study and Hauck's could be due to the variation in the doses of parasites used. In this study, paromomycin successfully showed its antihistomonal effect with the same doses of histomonads used in the tiamulin test. Therefore, the finding of this study was likely reliable. 
In general, the effect of tiamulin in the prevention and treatment of histomoniasis is still a controversial issue. Its antihistomonal effect was reported in an in vitro experiment by Hauck (2010), while van der Heijden (2011) claimed the ineffectiveness of the drug in another test. The main difference between these two studies was the dose of the parasites, which probably explained the contradictory results obtained.

\section{Arsenical Compounds}

Nitarsone (4-nitrophenylarsonic acid), a pentavalent arsenical compound, was at one time the only available drug on the U.S. market for the purpose of preventing histomoniasis. Unfortunately, this drug was withdrawn by the U.S. Food and Drug Administration at the end of 2015 (Administration USFaD, 2015). There have been various studies assessing the effectiveness of this chemical and its commercial form, Histostat-50, on preventing blackhead disease.

In 2004, scientists from the research team led by Duffy (2004) compared the antihistomonal activity of dietary Natustat with Histostat, a commercial form of nitarsone. Six hundred oneday-old chickens were divided into four groups of 150 and then subjected to different treatments. The first group was the control in which birds were neither infected with histomonads nor supplemented with Natustat or Histostat. In the second group, the birds were given histomonads but not treatment drugs. Nitarsone at 0.1875 $\mathrm{kg} /$ Tone was supplemented to the birds in group 3 , whereas Natustat at $1.925 \mathrm{~kg} /$ Tone was given to chickens in group 4 . Then the birds in both groups were experimentally infected with histomonads. The infection of histomonads was induced by using litter with a confirmed presence of Heterakis gallinarum infected by $H$. meleagridis. The results of this study illustrated that the birds treated with nitarsone had significantly lower cecal and liver lesion scores compared to those of the infected-non-treated group, indicating the antihistomonal activity of this arsenic compound (Duffy et al., 2004). Only one year later, the authors carried out a similar study, but this time in male turkeys. Once again, dietary Natustat for the control of $H$. meleagridis was assessed by comparing the effectiveness of this plant substance product with that of nitarsone. The results obtained from this study showed that nitarsone substantially improved feed conversion ratios and reduced caecal and liver lesion scores in the nitarsone-supplemented turkeys compared with the infected nonsupplemented groups. The average body weight at 42 days was greater in the nitarsone supplemented group than in the infected nonsupplemented group (Duffy et al., 2005). The findings of these two studies are not strongly supported by persuasive evidence, as the major limitation of these experiments was the unconvincing design. The authors applied a natural method of histomonad infection that had not been tested rigorously by previous researchers. The authors neither described this method in detail nor cited its referencing source. According to Lund et al. (1957) fewer than 0.5\% of embryonated Heterakis gallinae eggs were found to harbor $H$. meleagridis infection, and more than $50 \%$ of the worms contained no eggs with Histomonas spp. Given that using litter containing $H$. meleagridis-infected $H$. gallinarum to introduce the infection is not a proven method, the findings of these studies require further confirmation.

Recently, Abraham et al. (2014) evaluated the sensitivity of $H$. meleagridis to nitarsone in culture media and turkeys. The three strains collected from different outbreaks were subjected to nitarsone in culture media. The results of the in vitro test showed that the strain of $H$. meleagridis obtained from the outbreak in Northern California had sustained resistance to nitarsone at $100 \mathrm{ppm}$. In the in vivo experiments, no significant differences between the nitarsonetreated and the control group were found when comparing weight gain and caecal and liver lesions scores. This study illustrates that a strain of $H$. meleagridis has obtained partial resistance to nitarsone. The acceptable sample size and strong trial control design are supportive of the results of this study. These findings have raised concerns from scientists about the resistance of this parasite to the only permitted antihistomonal drug.

Following the ban of certain arsenic compounds, scientists began searching for an 
alternative that could protect birds from histomoniasis. This search focused on the drugs available for poultry. Various drugs were tested in vitro, but not many of them successfully showed antihistomonal activity (Table 2). Several nitroimidazoles showed their effectiveness in the protection of birds from parasites (Table 2). However, they were banned in the E.U. and the U.S. due to the concern of carcinogenicity. Histostat-50, the commercial form of nitarsone, is the only arsenic compound that has shown potential effectiveness in preventing the disease. Recently, a strain of $H$. meleagridis was found to be partially resistant to this arsenic compound, which raised the concern of drug resistance to this unique drug. Among the various antibiotics tested, only paromomycin was capable of protecting turkeys from histomonad infection by reducing the mortality and the severity of pathological lesions, however, the use of this drug is tainted by the concern of antimicrobial resistance. The antihistomonal effect of tiamulin was still controversial since it showed a dose-reliant efficacy in in vitro experiments.

In brief, Histostat-50 and paromomycin are the two drugs that have been used in the U.S. for the prevention of histomoniasis in birds but there are certain concerns about the use of these drugs due to drug resistance and human safety. Histostat was already removed from the market by the U.S. Food and Drug Administration at end of 2015 due to its carcinogenic property.

\section{Plant extracts}

As chemotherapeutics against $H$. meleagridis were banned in the European Union in 2003, farmers have been left without an effective treatment against blackhead disease. In the search for alternative treatments, researchers have investigated the use of natural plant-based products.

Natustat is a natural, plant-derived proprietary product containing yeast-derived mannanoligosaccharide, organic mineral nutrients, and plant extracts, and is used as an additive to feed for poultry (Duffy et al., 2005). Various scientists have investigated the prophylactic effect of this herbal product against histomonad infections. The ability of Natustat to manipulate the composition of the intestinal microflora was proved by using PCR-DGGE techniques in the study of Waters et al. (2005). In the caecal contents of turkeys treated with Natustat, there were various probiotic species such as Lactobacillus spp. and Bifidobacterium spp., which may contribute to the success of this plant-derived product in the control of histomonad infection.

The antihistomonal effect of Natustat was assessed in the study of Duffy et al. (2004),

Table 2. Literature evaluating the antihistomonal effects of chemotherapeutic drugs

\begin{tabular}{|c|c|c|c|}
\hline \multirow{2}{*}{ Product } & \multirow{2}{*}{ In vitro } & \multicolumn{2}{|c|}{ In vivo (host species) } \\
\hline & & Chicken & Turkey \\
\hline $\begin{array}{l}\text { Diloxanide furoate, albendazole, mebendazole, } \\
\text { quinolone, carbadox }\end{array}$ & $\begin{array}{l}\text { Hu \& McDougald } \\
(2004)^{*}\end{array}$ & & \\
\hline $\begin{array}{l}\text { Tinidazole, metronidazole, ornidazole, } \\
\text { dimetridazole }\end{array}$ & $\begin{array}{l}\text { Hu \& McDougald } \\
\qquad(2004)^{\star \star}\end{array}$ & $\begin{array}{l}\text { Hu \& McDougald } \\
\qquad(2004)^{\star \star}\end{array}$ & \\
\hline Paromomycin sulfate & $\begin{array}{l}\text { Hu \& McDougald } \\
(2004)\end{array}$ & $\begin{array}{l}\text { Hu \& McDougald } \\
\qquad(2004)^{*}\end{array}$ & $\begin{array}{c}\text { Hafez et al. (2010); Bleyen } \\
\text { et al. (2010); } \\
\text { van der Heijden (2011) }\end{array}$ \\
\hline Histostat- $50^{\circledR}$ (nitarsone) & & Duffy et al. (2004) & $\begin{array}{l}\text { Duffy et al. (2005); } \\
\text { Abraham et al. (2014) }\end{array}$ \\
\hline
\end{tabular}

Note: * Proved ineffective for the protection of birds; ${ }^{* *}$ Banned in the E.U. and the U.S. 
which was evaluated in the section about arsenic compounds of this review. Among the different treatment groups, the chicks fed with Natustat had the heaviest average body weight, highest food conversion ratio, and lowest mortality. Further, the caecal lesion scores were significantly lower than in the nitarsone treated group. In a similar experiment performed in turkeys, Natustat fed birds had the same average body weights as the control (non-infected, nonsupplemented) birds (Duffy et al., 2005). Although the authors claimed that Natutstat was effective for the prevention of histomoniasis in chickens and turkeys, the reliability of these papers could be questioned due to their unconvincing study designs.

Hauck et al. (2007) investigated the effect of Natustat in histomonad-infected turkeys. Three of eight birds in each cage were infected intracloacally. Heavy paper was used to cover the floor for one week in order to spread the infection to the other five birds. In this study, turkey poults received the Natustat at different rates $(250 \mathrm{~g}$, $500 \mathrm{~g}$, or $1000 \mathrm{~g}$ per ton of feed). The results revealed that treatments with Natustat at doses higher than $250 \mathrm{~g}$ per ton yielded a significant reduction in caecal and liver lesion scores, and an increase in weight gain in indirectly exposed birds. The authors concluded that Natustat was effective in controlling the spread of histomoniasis within the flock. However, it was noticed that there was a significant difference in terms of mortality and weight gain between the directly infected and indirectly infected group, indicating unsuccessful indirect infections. These findings did not agree with the previous study conducted by McDougald \& Fuller (2005) where the authors proved the direct transmission of histomoniasis among birds.

Zenner et al. (2003) carried out an in vitro experiment testing the antiparasitic activity of three essential oil extracts made from fresh cinnamon leaves, lemon pericarp, and cloves of garlic against $H$. meleagridis. A dose of $10^{6}$. histomonads $\mathrm{mL}^{-1}$ was used for the anaerobic incubation, followed by the addition of different dilutions of the oils to determine the minimal lethal concentration (MLC). The synergistic action of the extracts was also analyzed. The results of this experiment showed that the three tested oils had an in vitro antihistomonal activity despite the absence of synergy among them. A consistent experimental design, including an acceptable concentration of incubation $\left(10^{6}\right.$ parasites $\mathrm{mL}^{-1}$ ), the determination of the MLC, and the quantification of live parasites in culture made the findings of this study reliable.

Three years later, Hafez \& Hauck (2006) continued their interest with these essential oils by conducting an in vivo study where the preventive efficacy of Protophyt, a herbal product containing essential oils of cinnamon, garlic, lemon and rosemary, against $H$. meleagridis infection was assessed. The authors divided 60 two-week-old turkey poults into three groups in a controlled trial. Group 1 was provided Protophyt as a feed additive 6 days prior to infection until the end of the experiment. Groups 2 and 3 remained untreated. At 3 weeks of age, the poults of Groups 1 and 2 were infected intracloacally with $147,500 \mathrm{H}$. meleagridis per bird. Lesion scores of the dead birds and euthanised birds were recorded. Real-time PCR was used to confirm the presence of histomonad DNA in the subjected birds. The results obtained in the study showed a $20 \%$ and $50 \%$ mortality rate in Groups 1 and 2, respectively, while no deaths occurred in Group 3. In addition, lesions were found only in caeca and liver, which is typical for histomonad infection. This study concluded that Protophyt was an effective alternative for the reduction of mortality in turkeys caused by histomoniasis. In this paper, the authors used a clear study design (control trial), an acceptable sample size (3 groups of 20 birds each), and a reliable statistic method ( $\mathrm{t}$ test). Also, a molecular technique with ensured specificity (real-time PCR) was applied to confirm the presence of the parasite in the subjected birds, which made these findings persuasive.

van der Heijden \& Landman (2008a) performed a study to examine the in vitro effect of four herbal products against $H$. meleagridis, namely Aromabiotic, Enteroguard, Photophyt B (drinking water additive), and Photopgyt SP (feed additive). A $H$. meleagridis strain was cultured in modified Dwyer medium prior to the 
subculture. Dimetridazole and Histostat-50 were used as positive controls, while PBST was used as a negative control. Photophyt B at $0.24 \%$ caused a gradual decrease in the number of parasites over time. By using Enteroguard suspended at $400 \mu \mathrm{g} \mathrm{mL}{ }^{-1}$, the decline in the number of histomonads was observed from $48 \mathrm{~h}$. A significant lower parasite count in comparison with the negative control was obtained only after $72 \mathrm{~h}$ of incubation. Aromabiotic and Photopgyt SP showed no inhibitory effects on histomonad growth. In this in vitro experiment, the antihistomonal effects of the herbal products were not guaranteed since the growth of $H$. meleagridis can be inhibited only if the products were bactericidal to the caecal bacteria present in the culture of $H$. meleagridis. Using an antibiotic induced a complete inhibition of caecal bacterial growth that resulted in the inhibition of histomonads in this study. Moreover, the intestinal flora consisted of various species. A product may significantly destroy one or more bacterial species that are essential for histomonad growth. For these reasons, the results of this study are not reliable, and might need confirmation in an in vivo study.

Following the in vitro experiment, the authors performed an in vivo experiment with the same strain of $H$. meleagridis to confirm the antihistomonal activity of Enteroguard and Protophyt. Van der Heijden \& Landman (2008b) conducted the experiment using 234 two-weekold turkeys divided into 13 groups $(\mathrm{n}=18)$. The birds were either inoculated with 100,3162 , or 200,000 histomonads per bird. The negative control group was sham inoculated while the positive control was provided with no feed additives, Dimetridazole or Histostat-50. The study resulted in a mortality rate of $100 \%$ in the birds inoculated with 200,000 histomonads in all groups except the Dimetridazole-fed group. This study concluded that Protophyt and Enteroguard were ineffective at protecting birds from histomonad infection at a dose of 200,000 parasites/bird. The results obtained are convincing since the authors had designed a typical controlled trial with sufficient group sizes to justify statistical significance. Moreover, the authors used an adequate range of infection rates.
The method of intracloacal inoculation used in the experiment was likely more reliable in terms of avoiding re-infection as found in cases of using litter direct lateral transmission. However, it is necessary to revise the virulence of the strain and the dose used in this experiment. The dose of 200,000 histomonads per bird caused mortality in the group treated with Dimetridazole and $100 \%$ mortality in the group of Histostat-50 although these products had been reported as highly effective drugs against Histomonas spp. (Hu \& McDougald, 2004).

Grabensteiner et al. (2008) performed a study to investigate the in vitro and in vivo antihistomonal activities of 43 plant substances from 18 organic wastes obtained from the food industry. During the in vitro experiment, only four ethanolic extracts completely inhibited the growth of $H$. meleagridis in the culture with an initial inoculation of 100,000 histomonads $\mathrm{mL}^{-1}$, namely the ethanol extracts of saw palmetto, thyme, pumpkin fruit, and the water and ethanol extract of grape seed. These four extracts were selected for the subsequent in vivo experiment. The concentration of the extracts administered to the birds was increased from $0.1 \%$ to $1 \%$ during the experiment. The birds were experimentally infected with $10^{3}$ histomonads/bird via cloaca. All four tested extracts showed only positive effects of delaying mortality for up to 18 days in comparison to the control group. The authors concluded that none of the tested substances were able to prevent Histomonas spp. infection in turkeys. These findings are strongly supported by an explicit control trial study with a reliable experimental method and valid in vitro and in vivo test system. In addition, all data were analyzed by reliable statistic software.

Responding to the demand of an antihistomonal alternative, various plant-derived products have been tested both in vitro and in vivo. Several plant products successfully inhibited the growth of $H$. meleagridis in culture media such as Enteroguard and several ethanolic extracts (Table 3). However, none of these demonstrated dependable protection for birds from histomoniasis in an in vivo environment. In three experiments, Natustat showed its effectiveness against histomoniasis in birds 
(Table 3). The fact that these studies used infective methods made the results controversial. Therefore, it is recommended that further research should be conducted to evaluate the effects of Natustat using different modes of parasitic infection.

Similarly, controversies about the effects of Protophyt were found among the studies. This is one of a very few plant products that has shown an antihistomonal effect. It would be worthwhile for researchers to conduct further studies on this product.

\section{Vaccinations}

While chemotherapeutical products for the control of blackhead disease were used under high restrictions, and none of the plant-based substances demonstrated consistent protection for birds against the disease, vaccination became a compelling interest for scientists in the quest to discover an effective treatment and prevention for histomoniasis.

In 2008, Hess et al. (2008) investigated the efficacy of vaccines for the protection of turkeys from blackhead disease. The authors tested both an inactivated vaccine and an attenuated vaccine. The former was obtained through a freezing and thawing process and the addition of formol to a $H$. meleagridis clonal culture, while the latter was achieved by passaging the cloned parasites for around 2 years in intervals of 2-3 days. In this experiment, $0.5 \mathrm{~mL}$ of inactivated vaccine was intramuscularly injected, whereas the live vaccine was administered cloacally. The vaccinated birds were subjected to a challenge of 10,000 parasites/bird intracloacally. The turkeys that received the inactivated vaccine developed clinical signs and subsequently died, which indicated that the vaccine did not confer adequate protection. The birds that received the attenuated vaccine did not show any clinical signs specific for histomoniasis. In addition, no parasite DNA was recovered from the livers of the vaccinated birds, indicating that the vaccine successfully protected the birds.

An immunological study by Bleyen et al. (2009) was carried out to evaluate the effects of active and passive immunity in the protection of turkeys from histomoniasis. Active immunization was obtained by directly injecting

Table 3. Literature evaluating antihistomonal effect of plant substrate products

\begin{tabular}{|c|c|c|c|c|}
\hline \multirow{2}{*}{\multicolumn{2}{|c|}{ Product }} & \multirow{2}{*}{ In vitro } & \multicolumn{2}{|r|}{ In vivo (host species) } \\
\hline & & & Chicken & Turkey \\
\hline \multicolumn{2}{|l|}{ Aromabiotic } & $\begin{array}{l}\text { van der Heijden \& } \\
\text { Landman }(2008 \mathrm{a})^{*}\end{array}$ & & $\begin{array}{l}\text { van der Heijden \& Landman } \\
\qquad(2008 b)^{*}\end{array}$ \\
\hline \multicolumn{2}{|l|}{ Enteroguard } & $\begin{array}{l}\text { van der Heijden et al. } \\
\text { (2008a) }\end{array}$ & & van der Heijden et al. (2008b)* \\
\hline \multicolumn{2}{|l|}{ Natustat } & & $\begin{array}{l}\text { Duffy et al. } \\
\quad(2004)\end{array}$ & $\begin{array}{l}\text { Duffy et al. (2005); Waters et al. } \\
\quad \text { (2005); Hauck et al. (2007) }\end{array}$ \\
\hline \multirow{2}{*}{$\begin{array}{l}\text { Protophyt } \\
\text { (Essential oils of cinnamon, } \\
\text { garlic, lemon, and rosemary) }\end{array}$} & $\begin{array}{l}\text { Protophyt } \\
\text { B }\end{array}$ & $\begin{array}{l}\text { van der Heijden et al. } \\
\qquad(2008 a)\end{array}$ & & van der Heijden et al. (2008b)* \\
\hline & $\begin{array}{l}\text { Protophyt } \\
\text { SP }\end{array}$ & $\begin{array}{l}\text { van der Heijden et al. } \\
\qquad(2008 a)^{*} \\
\text { Zenner et al. (2003) }\end{array}$ & & $\begin{array}{c}\text { Hafez \& Hauck (2006); Van der } \\
\text { Heijden et al. }(2008 b)^{*}\end{array}$ \\
\hline \multicolumn{2}{|c|}{ Ethanol extracts of saw palmetto } & $\begin{array}{c}\text { Grabensteiner et al. } \\
(2008)\end{array}$ & & Grabensteiner et al. (2008)* \\
\hline \multicolumn{2}{|c|}{ Ethanol extracts of thyme } & $\begin{array}{c}\text { Grabensteiner et al. } \\
\text { (2008) }\end{array}$ & & Grabensteiner et al. (2008) \\
\hline \multicolumn{2}{|c|}{ Water and ethanol extracts of grape seed } & $\begin{array}{c}\text { Grabensteiner et al. } \\
(2008)\end{array}$ & & Grabensteiner et al. (2008) \\
\hline \multicolumn{2}{|c|}{ Ethanol extracts of pumpkin fruit } & $\begin{array}{c}\text { Grabensteiner et al. } \\
(2008)\end{array}$ & & Grabensteiner et al. (2008) \\
\hline
\end{tabular}

Note: * Product was confirmed ineffective. 
H. meleagridis antigens intramuscularly or by experimental infection via the cloaca, while passive immunization was implemented by intraperitoneal administration of antisera from immunized birds. These antisera were previously assessed for their capacity to destroy $H$. meleagridis. In addition, the transfer of antisera was assessed by measuring titers of anti- $H$. meleagridis antibodies. A strong antiparasitic serum antibody response was observed in both active immunization groups. On the contrary, the birds were not protected against a challenge with $3 \times 10^{5}$ histomonads in the passive immunized group despite the successful transfer of antiparasite antibodies. This indicated that passive immunization by transferring serum antibodies did not have a protective effect in turkeys.

In the study of Liebhart et al. (2010), the efficacy of oral vaccination of turkeys using attenuated $H$. meleagridis was evaluated. The authors also assessed the negative effects of this vaccine prototype on the performance of the birds. Five different groups of birds were used for the experiments, namely cloacally challenged 2 weeks after being orally vaccinated, cloacally challenged 4 weeks after being orally vaccinated, non-vaccinated but inoculum challenged, vaccinated but not challenged, and nonvaccinated not-challenged. The results of this experiment showed that the birds that were challenged 4 weeks after vaccination, as well as the vaccinated but not-challenged and the nonvaccinated not-challenged groups, did not show any clinical signs related to histomoniasis. All the birds in the non-vaccinated group and $71.4 \%$ of the birds cloacally challenged 2 weeks after vaccination contracted blackhead disease. Moreover, none of the vaccinated but notchallenged birds showed any abnormal performance. These findings not only supported the claim of Hess et al. (2008) about the effectiveness of the attenuated vaccine against $H$. meleagridis but also proved that the vaccine could be administered orally (Liebhart et al., 2010).

A study by Liebhart et al. (2010) evaluated the effects of virulent and attenuated $H$. meleagridis on turkeys and chickens. The virulent histomonads were obtained from clonal culture after 21 passages, while the attenuated vaccine was achieved after 295 passages. The turkeys were subjected to either virulent histomonads or attenuated histomonads with a dose of $10^{4}$ parasites administered orally, whereas the chickens were challenged with a similar infection regime and an additional cloacal dose. The results showed that liver and caecal lesions were found in both the chickens and turkeys receiving virulent infections, while no changes were observed in the birds infected with attenuated parasites. Moreover, the virulent parasites were found in several organs of the birds, whereas the attenuated isolates were present only in caecal samples in the PCR test. This indicated that attenuated $H$. meleagridis did not cause any adverse effects to infected chickens and turkeys. This was one of a very few studies focusing on vaccinations of this parasite in chickens. These findings could be useful for further investigations in the vaccine development for histomoniasis. However, the design of this study was not strongly convincing. The authors used a dose of only $10^{4}$ histomonads per bird, which was significantly lower than the dose used by other scientists, ranging from $1.4 \times 10^{5}$ to $10^{6}$ parasites per bird (Zenner et al., 2003; Hu \& McDougald, 2004; Hafez \& Hauck, 2006; van Der Heijden et al., 2008). It could explain why no changes were observed in the birds infected by attenuated parasites. Further studies are required to confirm the effectiveness of the immunization produced by these attenuated isolates.

A recent study conducted by Nguyen Pham et al. (2013) examined the protective effectiveness of the low-virulent $H$. meleagridis against blackhead disease in turkeys. The lowvirulent $H$. meleagridis was produced by serial intracloacal passages, which are also known as back passages. The back passage comprised of an intracloacal inoculation of histomonad to 3week-old birds. Thirteen days post-inoculation, these birds were euthanized to obtain the parasite for reinoculation of new birds. An examination of the virulence of the back passages showed that the last three intracloacal passages, did not cause any of the typical lesions found in turkeys. Subsequently, back passage 10 was used to test the protective capacity against $H$. meleagridis. 
The birds vaccinated with this low virulent back passage yielded minor clinical signs, and lower liver and caecal lesion scores compared to the unvaccinated challenged group; and no mortality compared to $71 \%$ in the unvaccinated group in an experimental challenge with a virulent strain of the parasite. This result indicated that the lowvirulent $H$. meleagridis attenuated by serial intracloacally passages was able to protect turkeys from a virulent strain of this parasite. A logical experiment design used in this study made its findings persuasive.

In general, most studies evaluating histomoniasis vaccination were conducted exclusively in turkeys. The transfer of serum antibodies from experimentally infected birds was unable to protect birds from histomonads infection, while active immunization created by injecting antigens showed protective activity. Several authors proved that a vaccination with in vitro attenuated parasites could provide protection to turkeys against $H$. meleagridis independently of administration (Table 4). Additionally, this type of vaccination did not alter the performance of the subjected birds. In a practical sense, the production of an effective vaccine would require not only sophisticated techniques but also a harmonization between laboratory experiments and field demand.

\section{The future of histomoniasis treatments}

Following the ban of nitroimidazoles, recognized as high potential antihistomonal drugs in the European Union and some other countries, due to the concern of ecological and human health threats, researchers were forced to search for viable alternatives in preventing histomoniasis. In general, three different approaches were investigated to deal with the situation. These were the application of antibiotics showing antiprotozoal activity, testing plant substances, particularly some essential oils, and the attempt to develop effective vaccines.

Among the various drugs tested, Histostat50 and Paromomycin are the two available drugs in some markets that possess antihistomonal activity despite recently raised concerns about deleterious side effects such as bacterial resistance and consumer safety. It is, therefore, crucial for policymakers to strike a balance between the benefits of the consumer and the sustainability of animal production.

A large number of plant substances were tested for their effectiveness against histomoniasis, none of which conferred reliable protection to birds in in vivo experiments. There was a lack of standardized methodology for infection among the studies that led to inconsistent results. It is therefore recommended to address this issue in further investigations.

Approaches to different types of immunizations showed that only vaccinations with attenuated parasites could induce reliable protection from histomoniasis in turkeys. However, the production of vaccines, particularly for this protist, would be challenging due to a high level of sophisticated techniques required.

\section{Acknowledgements}

We are grateful for the comments of Dr. Anne Beasley at the University of Queensland. She also answered numerous questions about the language of this paper. We also thank Prof. Nguyen Van Tho and Dr. Nguyen Thi Hong Chien, our colleagues at the Vietnam National University of Agriculture, who also looked over our transcriptions and provided valuable comments.

Table 4. Literature evaluating the effectiveness of vaccinations in the protection of birds from histomoniasis

\begin{tabular}{|c|c|c|c|}
\hline \multirow[t]{2}{*}{ Passive vaccination } & \multicolumn{3}{|c|}{ Active vaccination } \\
\hline & Antigens & Inactivated parasite & Attenuated parasite \\
\hline Bleyen et al. (2009)* & Bleyen et al. (2009) & Hess et al. $(2008)^{\star}$ & $\begin{array}{c}\text { Hess et al. (2008); } \\
\text { Liebhart et al. (2010); } \\
\text { Nguyen Pham et al. (2013) }\end{array}$ \\
\hline
\end{tabular}

Note: * Proved to be ineffective to protect birds from blackhead disease. 
We acknowledge the insightful comments offered by the anonymous reviewers and the hard work of the language reviewers who saved us from many errors.

\section{References}

Abraham M., McDougald L. R. \& Beckstead R. B. (2014). Blackhead disease: Reduced sensitivity of Histomonas meleagridis to nitarsone in Vitro and in Vivo. Avian diseases. 58(1): 60-63.

Administration USFaD. FDA. (2015). Announces Pending Withdrawal of Approval of Nitarsone. Retrieved from https://www.ngfa.org/news/feed-news/fda-announcespending-withdrawal-of-nitarsone-for-use-in-turkeyschicken/\#: :text=The\%20Food\%20and\%20Drug\%20 Administration,by\%20the\%20end\%20of\%202015 on October 10, 2019.

Araújo J. L., Olinda R. G., Frade M. T. S., Maia L. Â. \& Dantas A. F. M. (2015). Histomoniasis outbreak in free-range chickens in semiarid Paraíba, Brazil. Semina: Ciências Agrárias. 36(1): 307-312.

Bleyen N., De Gussem K., Nguyen Pham Anh Dao, Ons E., Van Gerven N. \& Goddeeris B. M. (2009). Noncurative, but prophylactic effects of paromomycin in Histomonas meleagridis-infected turkeys and its effect on performance in non-infected turkeys. Veterinary Parasitology. 165(3-4): 248-255.

Bleyen N., Ons E., De Gussem M. \& Goddeeris B. M. (2009). Passive immunization against Histomonas meleagridis does not protect turkeys from an experimental infection. Avian Pathology. 38(1): 71-76.

Byrne D. (2001). Commission Regulation (EC) No. 2205/2001 of November 14, 2001 amending Council Directive 70/524/EEC concerning additives in feedingstuffs as regards withdrawal of the authorisation of certain additives 2001. Retrieved from https://eur-lex.europa.eu/legal-

content/EN/TXT/?uri=CELEX\%3A32001R2205 on October 10, 2019.

Cushman S. (1893). The production of turkeys. Rhode Island Agricultural Experiment Station Bulletin. 25: 89-123.

Duffy C. F., Sims M. D. \& Power R. F. (2005). Evaluation of dietary Natustat for control of Histomonas meleagridis in male turkeys on infected litter. Avian Diseases. 49(3):423-425.

Duffy C. F., Sims M. D. \& Power R. F. (2004). Preliminary evaluation of dietary natustat ${ }^{\mathrm{TM}}$ versus histostat ${ }^{\circledR}$ (Nitarsone) for control of Histomonas meleagridis in broiler chickens on infected litter. International Journal of Poultry Science. 3(12): 753-757.

EFSA (2009). Scientific Opinion of the Panel on Additives and Products or Substances used in Animal Feed (FEEDAP) on a request from the European
Commission on the preliminary evaluation of the safety and efficacy of paromomycin sulphate for turkeys for fattening and turkeys reared for breeding. The EFSA Journal. 1095: 1-22.

Esquenet C., De Herdt P., De Bosschere H., Ronsmans S., Ducatelle R. \& Van Erum J. (2003). An outbreak of histomoniasis in free-range layer hens. Avian Pathology. 32(3): 305-308.

Grabensteiner E., Liebhart D., Arshad N. \& Hess M. (2008) Antiprotozoal activities determined in vitro and in vivo of certain plant extracts against Histomonas meleagridis, Tetratrichomonas gallinarum and Blastocystis sp. Parasitology Research. 103(6): 12571264.

Grabensteiner E., Liebhart D., Weissenböck H. \& Hess M. (2006). Broad dissemination of Histomonas meleagridis determined by the detection of nucleic acid in different organs after experimental infection of turkeys and specified pathogen-free chickens using a mono-eukaryotic culture of the parasite. Parasitology International. 55(4): 317-322.

Hafez H. M., Schulze D., Hauck R. \& Lüschow D. (2005). Histomonas meleagridis: The Situation after the Ban of the Last Available Drug in the EU. Proceedings of the fifty-fourth Western pounltry disease conference. Vancouver, B.C., Canada.

Hafez H.M., Hauck R., Gad W., De Gussem K. \& Lotfi A. (2010). Pilot study on the efficacy of paromomycin as a histomonostatic feed additive in turkey poults experimentally infected with Histomonas meleagridis. Archives of Animal Nutrition. 64(1): 77-84.

Hafez H. M. \& Hauck R. (2006). Efficacy of a herbal product against Histomonas meleagridis after experimental infection of turkey poults. Archives of Animal Nutrition. 60(5): 436-642.

Hauck R., Armstrong P. L., Fuller L. \& McDougald L. R. (2007). The effect of Natustat ${ }^{\circledR}$ on Histomoniasis in turkeys. Proceedings of the 56th Western poultry disease . March 26-29, 2007. Las Vegas, Nevada. 102104.

Hauck R., Balczulat S. \& Hafez H. M. (2010). Detection of DNA of Histomonas meleagridis and Tetratrichomonas gallinarum in German poultry flocks between 2004 and 2008. Avian Diseases. 54(3): 1021-1025.

Hess M., Liebhart D., Grabensteiner E. \& Singh A. (2008). Cloned Histomonas meleagridis passaged in vitro resulted in reduced pathogenicity and is capable of protecting turkeys from histomonosis. Vaccine. 26(33): 4187-4193.

Hu J. \& McDougald L. R. (2004). The efficacy of some drugs with known antiprotozoal activity against Histomonas meleagridis in chickens. Veterinary parasitology. 121(3-4): 233-238.

Kawalek J. C., Carson M., Conklin S., Lancaster V., Howard K. \& Ward J. (2011). Provide data on various arsenic species present in broilers treated with 
roxarsone: comparison with untreated birds. Final report on Study 275.30. 2011. US Food and Drug Administration: Laurel, MD, USA.

Kempf I., Le Roux A., Perrin-Guyomard A., Mourand G., Le Devendec L., Bougeard S., Richez P., Le Pottier G. \& Eterradossi N. (2013). Effect of in-feed paromomycin supplementation on antimicrobial resistance of enteric bacteria in turkeys. The Veterinary Journal. 198(2): 398-403. DOI: 10.1016/j.tvj1.2013.05.030.

Liebhart D., Windisch M. \& Hess M. (2010). Oral vaccination of 1-day-old turkeys with in vitro attenuated Histomonas meleagridis protects against histomonosis and has no negative effect on performance. Avian Pathology. 39(5): 399-403.

Lund E. E. \& Burtner Jr R. H. (1957). Infectivity of Heterakis gallinae eggs with Histomonas meleagridis. Experimental Parasitology. 6(2):189-93. DOI: 10.1016/0014-4894(57)90014-0.

McDougald L. R. \& Fuller L. (2005). Blackhead disease in turkeys: direct transmission of Histomonas meleagridis from bird to bird in a laboratory model. Avian Diseases. 49(3): 328-331.

McDougald L. R. \& Hu J. (2001). Blackhead disease (Histomonas meleagridis) aggravated in broiler chickens by concurrent infection with cecal coccidiosis (Eimeria tenella). Avian Diseases. 45(2): 307-312.

Nguyen Duc Tan, Bilic I., Jaskulsa B., Hess M. B., Le Duc Quyet, Le Hua Ngoc Luc, Huynh Vy Vu, Nguyen Thi Sam \& Vu Khac Hung (2015). Prevalence and genetic characterization of Histomonas meleagridis in chickens in Vietnam. Avian diseases. 59(2):309-314. DOI: 10.1637/10964-102414-Reg.

Nguyen Pham Anh Dao., De Gussem J. K. \& Goddeeris B. M. (2013). Intracloacally passaged low-virulent Histomonas meleagridis protects turkeys from histomonosis. Veterinary Parasitology. 196(3): 307-313.

Reece R. L., Beddome V. D. \& Barr D. A. (1986). Diseases diagnosed in replacement layer and breeder chicken flocks in Victoria, Australia, 1977 to 1985 . Veterinary
Record. 119(19): 471-475.

Somkid K. P. K. \& Niwat C. (2006). Outbreak of histomoniasis in cross breed native chickens from one private farm in Nakorn Pathom province. Retrieved on October 18, 2019 from http://agris.fao.org/agrissearch/search.do?recordID=TH2006000328.

Swayne DEG. J. R., McDougald L. R., Nolan L. K., Suarez D. L. \& Nair V. (2013). Diseases of Poultry. 13, editor: John Wiley \& Sons, Inc; 1423.

van der Heijden H. M. J. F., De Gussem K. \& Landman W. J. M. (2011). Assessment of the antihistomonal effect of paromomycin and tiamulin. Tijdschrift Voor Diergeneeskunde. 136(6): 410-416.

van der Heijden H. M. (2009). Detection, typing and control of Histomonas meleagridis. Retrieved from http://dspace.library.uu.nl/bitstream/handle/1874/322 42/heijden.pdf?sequence $=1$ on October 18, 2019.

van der Heijden H. M. J. F. \& Landman W. J. M. (2008a). In vitro effect of herbal products against Histomonas meleagridis. Veterinary Parasitology. 154(1): 1-7.

van der Heijden H. M. \& Landman W. J. M. (2008b). In vivo effect of herbal products against Histomonas meleagridis in turkeys. Avian Pathology: journal of the WVPA. 37(1): 45-50.

Waters S. M., Duffy C. F. \& Power R. F. G. (2005). PCRDGGE analysis of caecal microflora of Natustat ${ }^{\mathrm{TM}}$ supplemented turkeys challenged with Histomonas meleagridis. International Journal of Poultry Science. 4(9): 620-627.

Zahoor M. A., Liebhart D. \& Hess M. (2011). Progression of histomonosis in commercial chickens following experimental infection with an in vitro propagated clonal culture of Histomonas meleagridis. Avian Diseases. 55(1): 29-34. DOI: 10.1637/9508-082110-Reg.1.

Zenner L., Callait M. P., Granier C. \& Chauve C. (2003). In vitro effect of essential oils from Cinnamomum aromaticum, Citrus limon and Allium sativum on two intestinal flagellates of poultry, Tetratrichomonas gallinarum and Histomonas meleagridis. Parasite. 10(2): 153-157. DOI: 10.1051/parasite/2003102153. 